\title{
MEJORANDO LA CALIDAD DE VIDA EN LA ENFERMEDAD DE PARKINSON DESDE LA MEDICINA BIORREGULADORA DE SISTEMAS, UNA NUEVA PERSPECTIVA, REPORTE DE CASO CLINICO.
}

\author{
Wilmer Jose Rojas Sanchez, ${ }^{1}$ Bibiana Maria Betancur Restrepo ${ }^{2}$ \\ Corresponding Author: Wilmer Jose Rojas Sanchez, wilmermedint@gmail.com \\ ${ }^{1}$ Especialista en Medicina Interna Universidad de los Andes Mérida - Venezuela, diplomado en \\ Medicina Integrativa y Bases Homeopáticas con Énfasis en Homotoxicología Universidad \\ Javeriana, internista Uce - Medellin, Colombia. | wilmermedint@gmail.com \\ ${ }^{2}$ Especialista en Pediatría Universidad de Antioquia, Medellin Colombia, diplomado en Medicina \\ Integrativa y Bases Homeopáticas con Énfasis en Homotoxicología Universidad Javeriana, pediatra \\ clínica Somer Medellin Colombia. | Bibipediatra@gmail.com
}

\begin{abstract}
Sabemos que la enfermedad de Parkinson, es el trastorno neurodegenerativo más frecuente, después de la enfermedad de Alzheimer y que su principal factor de riesgo es la edad avanzada. Dicha enfermedad supone cada vez más un reto de salud pública en nuestras sociedades occidentales progresivamente envejecidas.(1) Esta se caracteriza clínicamente por la presencia de la tríada motora: acinesia o lentitud de movimientos, temblor de reposo y rigidez, por ello ha sido clásicamente considerada y estudiada como un trastorno motor; Sin embargo, en las últimas décadas, ha aumentado el reconocimiento de manifestaciones no motoras como la apatía, el deterioro cognitivo o los síntomas disautonómicos entre otros.(1) Según la Asociación Europea de la Enfermedad de Parkinson el 94 \% de los pacientes sufre anomalías motoras, el $84 \%$ síntomas depresivos y en un $65 \%$ trastornos cognitivos, Estas diferencias fisiopatológicas están influenciadas por factores genéticos, emocionales y epigenéticos, que se expresan en una inflamación crónica de bajo grado ,asociado directamente a una mayor degeneración de las células neuronales ; ésta combinación supone para el paciente una progresiva disminución en su calidad de vida.
\end{abstract}

Se presenta el caso de un paciente de 78 años de edad con diagnóstico de enfermedad de Parkinson desde hace 17 años, en manejo médico convencional por especialistas en neurología, con pobre respuesta al tratamiento, con deterioro clínico y con alteración importante de su calidad de vida, que al momento de su valoración inicial, aplicando la escala de calidad de vida Euroqol 5D mostraba un puntaje $6.74 \%$, y en el termómetro de calidad de vida de esta misma escala 20 puntos \% , con una severidad según la escala Hoehn y Yahr tambien de calidad de vida , en estadio 5, indicando (necesita ayuda para todo permanece sentado o acostado), y por el índice de Katz que evalúa la independencia de un enfermo para realizar actividades de la vida diaria por calculadora virtual registrando los items obtenidos . 5 puntos ( incapacidad severa), se inicio tratamiento desde la medicina biorreguladora de sistemas, aplicando como conceptos 
la detoxificación básica y central, la inmunomodulación y la regulación del eje hipotálamo hipófisis adrenal (HHA) con medicamentos como Galium Heel, Cerecomp Compositum, Traumeel S , Neurexan entre otros. por vía oral y endovenosa; después de 3 meses de tratamiento se logra evidenciar en el paciente una mejoría notable de sus síntomas clínicos siendo muy evidente en el temblor, rigidez, agitación psicomotora, demostrado por cambios en los resultados de las escalas ; en la escala Euroqol 5D mejorando en un $62.43 \%$ en su calidad de vida , 50 puntos $\%$ en el termometro de calidad de vida de esta misma escala y ; pasando a un estadio 3 en la escala de Hoehn y Yahr (alteración bilateral leve o moderada con cierta inestabilidad postural), y obteniendo en el índice de Katz un puntaje de 3 puntos ; además se logro reducir la dosis de medicación antihipertensiva .

Con este caso clínico podemos sugerir que el uso de medicamentos biorreguladores en dosis bajas multiobjetivo y multicomponente favorecen el proceso de autorregulación de redes desreguladas en la Enfermedad de Parkinson como son el sistema linfático " glinfático", la red neuroendocrina ,mitocondrial y modulando la inflamación crónica de bajo grado; logrando disminuir la severidad de los síntomas, la limitación funcional y mejorando la calidad de vida .

Keywords: reporte de caso, medicina biorreguladora de sistemas, enfermedad de Parkinson, enfermedades neurológicas degenerativas, calidad de vida.

\section{Introduction}

La incidencia de la enfermedad de Parkinson en personas mayores de 60 años es de 13.4 por 100.000 habitantes/año en Estados Unidos, afecta en la actualidad de 4.1 a 4.6 millones de personas mayores de 50 años a nivel mundial y se calcula que para el 2030 esta cifra será duplicada.(2). Entre los factores de riesgo más ampliamente estudiados se encuentran los de tipo genético, hasta el año 2010 se habían relacionado 11 genes y 3 locus que aumentaban el riesgo de desarrollar la enfermedad, con un patrón autosómico dominante y autosómico recesivo, y frecuentemente relacionados con el inicio temprano de la enfermedad. El primer gen identificado fue el alfaSynuclein (SNCA) (PARK1), y la severidad de sus manifestaciones clínicas depende del número de repeticiones del gen. Así mismo se han descrito numerosos genes hasta el PARK16(3). Otros factores asociados al desarrollo de la enfermedad de Parkinson son el trauma craneoencefálico grave, alta ingesta de hierro, anemia crónica y trabajos de alta complejidad cognitiva; pero también la exposición a pesticidas, la residencia en el campo y la agricultura, como en el caso de nuestro paciente. La base fisiopatológica de la enfermedad de Parkinson es la degeneración en las neuronas dopaminérgicas, con la consiguiente disminución en la producción de dopamina. Se ha planteado que el daño en éstas células se asocia a disfunción mitocondrial, agregación de $\alpha$-sinucleina, alteración de la autofagia, estrés del retículo endoplasmático y la desregulación de la homeostasis intracelular de calcio, que genera una cascada apoptótica en las neuronas. Al generarse desinhibición de las neuronas estriatales debido al déficit de dopamina, se generan varios procesos que terminan en la desinhibición del núcleo subtalámico, lo que resulta en una disminución de la actividad locomotora y la consiguiente bradicinesia. También se genera una desinhibición de la actividad del tálamo, por lo que queda libre para enviar impulsos excitatorios a la corteza motora, lo 
cual genera temblor. Los pacientes también pueden presentar un grupo de síntomas no motores, explicados por la disfunción de neuronas no dopaminérgicas que incluyen degeneración de bulbo olfatorio, alteraciones del centro del sueño en el tronco cerebral y de los núcleos medulares que controlan funciones autonómicas, acumulación de cuerpos de Lewy en las estructuras límbicas y la neocorteza.(4) sin olvidar la asociación directa con depresión y ansiedad en los casos severos, impactando de forma importante la calidad de vida del paciente y de su familia. A pesar de los avances en su conocimiento, el diagnóstico de la enfermedad sigue siendo eminentemente clínico. No existe una prueba diagnóstica que confirme la sospecha clínica o permita hacer un seguimiento para su progresión, esto debe motivarnos a conocer la enfermedad, diagnosticarla y hacer el mejor enfoque terapéutico dado que se presume según las estadísticas que esta patología impactara de forma ascendente a nuestras sociedades en los próximos años.

\section{Timeline}

2019-11-13 - paciente que ingresa a la consulta de medicina interna con diagnostico de enfermedad de parkinson con importante deterioro en calidad de vida segun escalas de Euro qol, Yoehn y Yahr, y Katz, se propone manejo con medicina biorreguladora de sistemas.

\footnotetext{
2019-12-20 — asiste a control medico, termino detoxificación básica , inicio con detoxificación central, usando medicamentos como el Hypothalamus Suis Injeel, Cerecom Compositum, continuando por vía oral Neurexan y suplemento de magnesio
}

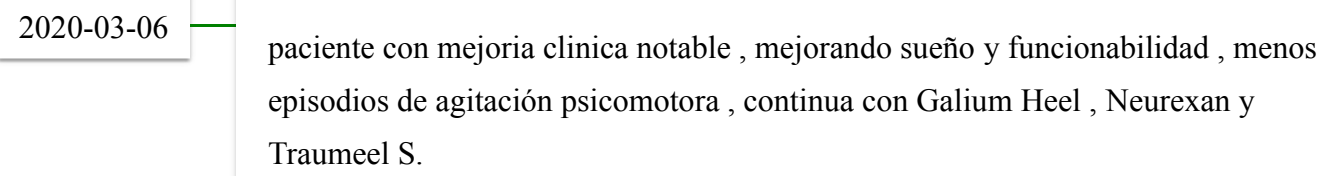
episodios de agitación psicomotora, continua con Galium Heel, Neurexan y Traumeel S.

2020-06-25 - asiste a control, refiriendo sentirse mucho mejor , mejorando calidad de sueño, temblor, con mejoría de sus índices en las escalas de euroqol D5, Yoehn y Yahr, kats , indicandose control cada 3 meses

$2020-12-03$ asiste a control, no hay nuevos deterioros clínicos, se coloco nueva sesión de medicina biorreguladora de sistemas con inclusión en este protocolo de Coenzyme Compositum y Ubichinon Compositum, se cita a control en 3 meses

\section{Narrative}


El paciente se atiende por primera vez en octubre de 2019, residente del municipio La Unión, Antioquia Colombia Casado con 12 hijos, agricultor en la juventud. Con enfermedad de Parkinson diagnosticada en el año 2004, se descartaron otras condiciones por neuroimagen. Además antecedente de osteoartrosis de rodillas, hipotiroidismo, enfermedad pulmonar obstructiva crónica por tabaquismo hasta hace 30 años, insuficiencia venosa de miembros inferiores, infarto de miocardio silente diagnóstico por ecocardiografía, tumor cutáneo, manejo con resección completa en 2018, bordes libres (Reporte histopatológico: Carcinona escamocelular in situ).

Recibía tratamiento con: Captopril $50 \mathrm{mg}$ cada 12 horas, metoprolol $50 \mathrm{mg}$ cada 12 horas, furosemida $40 \mathrm{mg}$ día, omeprazol $20 \mathrm{mg}$ día, Atorvastatina $40 \mathrm{mg}$ día, Carbonato de Calcio con Vitamina D 600/200 mg dia, Calcitriol 0,25 mg diarios, Acido Acetilsalicílico 100mg diarios, Levotiroxina $50 \mathrm{mcg}$ diarios, oxígeno suplementario por cánula nasal a 2 litros por minuto, 12 horas en la noche, nebulizaciones con bromuro de Ipratropio en solución cada 8 horas, Levodopa/Carbidopa tabletas de 25/250 mg 1 tableta am, $1 / 2$ al medio día, 1 en la tarde y $1 / 2$ en la noche, Quetiapina 25 mg día, Amantadina 100 mg cada 12 horas y Amitriptilina 25 mg cada día.

La familia refiere que el paciente no ha presentado mejoría con la medicación actual y con controles periódicos con neurología, incluso se quejan de deterioro dado por aumento en la rigidez, incapacidad para levantarse de la silla, para lo cual requiere de la ayuda de 2 personas; además de empeoramiento en los trastornos del sueño. Presenta insomnio de conciliación y despertares frecuentes, episodios de agitación psicomotora durante la noche y alucinaciones. Los episodios de agitación se presentan de manera intermitente, pero en ocasiones se pueden manifestar hasta 5 días seguidos. Se queja de dolor persistente en miembros inferiores, principalmente en las rodillas. Presenta incapacidad para la alimentación la cual debe ser suministrada por los familiares.

$\mathrm{Al}$ examen físico inicial presentaba los siguientes signos vitales: presión arterial 120/60 mmHg frecuencia cardiaca 83 latidos por minuto y frecuencia respiratoria 16 respiraciones por minuto No tenía adenomegalias cervicales, no dolor cervical a la movilización o palpación. A la exploración cardiopulmonar ruidos cardiacos rítmicos, murmullo vesicular disminuido de forma generalizada, sin ruidos agregados. Abdomen sin masas o megalias palpables. En el aspecto neurológico examen ocular normal, simetría facial con hipomimia, hipoquinesia y bradicinesia bilateral, temblor de reposo en ambas manos. Presentaba rigidez moderada en rueda dentada de predominio en hemicuerpo derecho. Marcha lenta sin balanceo de brazos. Marcha festinante. Sin signos piramidales No hay edema en miembros inferiores, deformidades en rodillas, ni dolor a la movilización de estas.

En la segunda consulta en enero de 2020, continúa con la medicación convencional habitual, pero con suplemento de oxígeno solo 8 horas cada noche y con bioterapia intravenosa ya para esta fecha habia terminado su sexta sesion de sueroterapia y continua Neurexan 2 tabletas sublingulaes diarias y suplemento magnesio $5 \mathrm{cc}$ oral cada 12 horas. La esposa refiere notable mejoría en la marcha y en la movilidad, con disminución de la rigidez, duerme mucho mejor y se han espaciado los episodios de agitación, los cuales se presentan ya una vez cada 15 días, indicándose en esta consulta disminuir dosis de antihipertensivo a la mitad por datos de hipotensión al interrogatorio y suspendiendo la suplementación de magnesio. 
Examen físico de esa consulta presión arterial 120/70 mmHg frecuencia cardiaca 90 latidos por minuto y frecuencia respiratoria de 17 respiraciones por minuto El paciente saluda, refiere sentirse bien. Presenta examen neurológico con movimientos oculares normales, simetría facial, leve hipoquinesia y bradicinesia bilateral, sin temblor en reposo en las manos. Aún se insinúa el signo de la rueda dentada de predominio derecho. Marcha lenta sin balanceo de brazos, pero con mejor patrón de movilidad. Sin signos piramidales. No se encuentra edema de miembros inferiores, deformidad en rodillas, ni dolor a la flexión de estas. Al terminar la consulta se levanta de la silla por sí solo, sin ayuda de sus familiares.

\section{ESTUDIOS DIAGNÓSTICOS}

Resonancia magnética cerebral Simple de 9-2-2018: atrofia encefálica de predominio frontal, leucoencefalopatía microangiopática leve, engrosamiento mucoso mastoideo bilateral.

Ecocardiograma transtorácico de 21-3-2019: fracción eyección 55 \% escara inferolateral en todos sus segmentos, acinesia inferior basal, resto de contractilidad normal.

Monitoreo ambulatorio de presión arterial (MAPA) en rango de normalidad, presión de pulso adecuada, presión arterial media 111/69.2 mmHg, promedio día 111/68.4 mmHg promedio noche $112 / 69 \mathrm{mmHg}$.

\section{9/12/2019: Vitamina D $32 \mathrm{ng} / \mathrm{Ml}$}

25/01/2020: paratohormona $59.4 \mathrm{pg} / \mathrm{mL}$, hemoglobina $18.9 \mathrm{Mg} / \mathrm{Dl}$ hematocrito $56.9 \%$ leucocitos 10.330 neutrófilos $69 \%$, plaquetas 192,000, TSH $4.92 \mathrm{mUI} / \mathrm{L}$ glucosa en ayunas $91 \mathrm{mg} / \mathrm{dl}$, potasio sérico $5.58 \mathrm{mEq} / \mathrm{L}$ calcio sérico $10 \mathrm{mg} / \mathrm{dl}$ fósforo inorgánico $3.8 \mathrm{mg} / \mathrm{dl}$ acido úrico $5.5 \mathrm{mg} \mathrm{dl}$, albúmina $4.6 \mathrm{~g} / \mathrm{dl}$ creatinina $1.16 \mathrm{mg} / \mathrm{dl}$, microalbuminuria $0.5 \mathrm{mg} / \mathrm{dl}$, uroanálisis no patológico.

\section{Perspective}

El paciente refiere "me siento mucho mejor". La esposa refiere "nos cambió la vida a todos"

\section{Discussion}

La enfermedad de Parkinson, si bien no es una enfermedad asociada a mortalidad directa o a disminución en la expectativa de vida, sí genera un marcado deterioro en la calidad de vida de los pacientes y su familia. Aquí presentamos el caso de un paciente de 78 años, con enfermedad de párkinson diagnosticada desde hace 17 años, con deterioro clínico acelerado el último año; Antes del inicio del tratamiento con medicina biorreguladora de sistemas presentaba compromiso marcado en calidad de vida, discapacidad física para la marcha y dependencia completa para autocuidado. Se encontró un estadio evolutivo de la enfermedad de 5 según la escala de calidad de vita de Hoehn y Yahr, en el índice de Katz 5 puntos ,que es el compromiso mas avanzado, Euroqol 5D : 6.74\% y termómetro de calidad de vida de la misma en 20 puntos 5 , a pesar del manejo con medicamentos tradicionales apropiados. Luego del tratamiento con medicina biorreguladora de sistemas vía intravenosa por sesiones de sueroterapia cada 8 días y en total 9 sesiones y suplementación temporal de magnesio y manteniendo Neurexan vía sublingual de forma permanente, se encontró una notable 
mejoría en la evolución del paciente, que se comenzó a notar incluso con regresión en su clasificación evolutiva de la enfermedad a 3 puntos según escalas de Hoehn y Yahr, y en el índice de Katz y mejoría en 50 puntos \% en termómetro de calidad de vida de Euroqol 5D con mejoría post tratamiento en un $62.46 \%$ por esta escala de Euroqol 5D ; ya con recuperación de su independencia para la mayoría de los procesos cotidianos. se recalca que al paciente no se le modifico en ningún momento su tratamiento por parte de neurología , pero si se logro disminuir dosis de su medicamento antihipertensivo .

haciendo el análisis el paciente recibió manejo con medicamentos que favorecen un proceso de detoxificación en sistema nervioso central y además soporte de órgano de las principales estructuras comprometidas en la enfermedad. se suministro, Galium Heel que favorece una detoxificación profunda, y ha sido tradicionalmente útil en enfermedades degenerativas crónicas del sistema nervioso, Cerecomp Compositum, Thalamus Compositum e Hypothalamus Suis Injeel, también con efecto de soporte mitocondrial. Dichos medicamentos contienen compuestos útiles en procesos espásticos, temblores, trastornos del sueño propios de la enfermedad, y también favorecen la recuperación de la matrix extracelular y de la función mitocondrial.

Aquí cabe mencionar que las neuronas dopaminérgicas se exponen constantemente al estrés oxidativo por varios mecanismos, entre ellos el metabolismo de la dopamina que puede ser por auto oxidación, dando como resultado la dopaminaquinona y radicales peróxido que se convierten en peróxido de hidrógeno o en radicales peroxinitritos los cuales aumentan la citotoxicidad. En los pacientes con enfermedad de Parkinson éste mecanismo esta acentuado debido a que hay mayor acumulación de dopamina en el citoplasma por el aumento en la producción y acumulación en el citoplasma y no en las vesículas como ocurre de manera fisiológica, además de que el envejecimiento celular conlleva a disminución de la actividad del glutatión que ayuda a metabolizar los radicales libres. Es importante realizar más estudios para determinar si los medicamentos que aumentan la dopamina pueden acelerar la destrucción de las neuronas dopaminérgicas al aumentar el estrés oxidativo. El magnesio que se administró al paciente es bien conocido por su rol en diferentes procesos celulares incluyendo la producción de energía, síntesis de proteínas, como cofactor de procesos enzimáticos, estabilización de membrana incluso mantenimiento del DNA y RNA. El selenio que se encuentra en el Cerecomp es un cofactor de múltiples selenoenzimas en especial la Glutatión Peroxidasa y la Selenoproteína P, las cuales son antioxidantes que cumplen un rol protector frente a la lipoperoxidación de las membranas celulares, lo cual está descrito como causa del daño de las neuronas dopaminérgicas en la enfermedad. Como mencionamos, en la fisiopatología de la enfermedad se describe un importante deterioro mitocondrial, lo cual se ha evidenciado en la disminución de la actividad del complejo I de la cadena transportadora de electrones, por lo que se considera que en esta enfermedad también hay dificultades con la producción energética celular, que finalmente termina en el daño de las células neuronales, de hay la importancia de seguir usando medicamentos de medicina biorreguladora que actúen para evitar la progresión de la degeneración celular, en ese contexto se decide modificar su ultima sesion de sueroterapia usando ya medicamentos importantes como son el Coenzyme Compositum y el Ubichinon Compositum no dudando de su impacto favorable en el mejoramiento de la matrix extracelular y en la producción energética mitocondrial que se debe restaurar en esta patología. 


\section{$\underline{\text { Conclusion }}$}

Con el aumento en la expectativa de vida de la población mundial, cada vez son más frecuentes las enfermedades como la Enfermedad de Parkinson, por esta razón es tan importante buscar opciones de terapias complementarias que ayuden a mejorar los síntomas y la calidad de vida de los pacientes y sus familias, en el caso de este paciente fue tangible la buena respuesta terapéutica, obtenida con el tratamiento de medicina biorreguladora de sistemas y que se demostró por los cambios obtenidos en las escalas de Euroqol 5D, termometro de calidad de vida de esta, en la escala de Hoen y Yahr y en el índice de Katz .,Seria de gran importancia realizar el diagnóstico de esta enfermedad en sus fases iniciales y con esto sería, posible, intervenir con medicina biorreguladora de sistemas de una forma más oportuna para realizar un tratamiento de neuroprotección temprana y evitar así el impacto nefasto que ocasiona esta enfemedad en la salud de quien la padece.

\section{$\underline{\text { Acknowledgements }}$}

Dra Deisy Hernandez

Dra Mónica Name

Dr Baltazar Guerrero

\section{$\underline{\text { References }}$}

1- Rev. Med. Clin. condes - 2016; 27(3) 363-379]

2- Arch Neurocien (Mex) INNN, 2014 Vol. 19 | No. 3 julio-septiembre 2014 | 157

3- Acta Neurol Colomb. 2014; 30(4):300-306

4- Revista de la Universidad Industrial de Santander. Salud Vol.50 No.1 Enero - Marzo de 2018. Enfermedad de Parkinson: fisiopatología, diagnóstico y tratamiento .

\section{Attachments}




\begin{tabular}{|c|c|c|c|c|c|c|c|c|}
\hline $\begin{array}{l}\text { Fecha } \\
\text { Medicamento }\end{array}$ & $12-11-19$ & 19-11-19 & $26-11-19$ & $3-12-19$ & $9-12-19$ & $17-12-19$ & $7-03-20$ & $25-06-20$ \\
\hline Neurexan & $x$ & $x$ & $x$ & $x$ & $x$ & $x$ & $x$ & $x$ \\
\hline Nux Vomica & $\mathrm{x}$ & & & & & & & \\
\hline Berberis homaccord & $x$ & & & & & & & \\
\hline Lymphomyosot & $x$ & & & & & & & \\
\hline Thalamus & & $x$ & $x$ & & & & & $\mathrm{x}$ \\
\hline Traumeel s & $x$ & $\mathrm{x}$ & $x$ & $x$ & $x$ & $\mathrm{x}$ & $x$ & $x$ \\
\hline Cerecomp & & $\mathrm{x}$ & $x$ & $\mathrm{x}$ & $x$ & $\mathrm{x}$ & $\mathrm{x}$ & $x$ \\
\hline Galium & & $\mathrm{x}$ & $x$ & $x$ & $x$ & $x$ & $x$ & \\
\hline Hypothalamus suis injeel & & & & $x$ & $\mathrm{x}$ & $x$ & $x$ & $x$ \\
\hline
\end{tabular}

Terapia de medicina biorreguladora suministrada según las fechas

se especifica por fechas las sesiones de tratamiento utilizándose 1 ampolla de cada uno de los medicamentos anotados vía endovenosa diluidos en 100 cc de solución salina al 0.9\%, aplicados por la técnica de sueroterapia ; Neurexan vía sublingual como medicación de uso diario 2 veces al día,

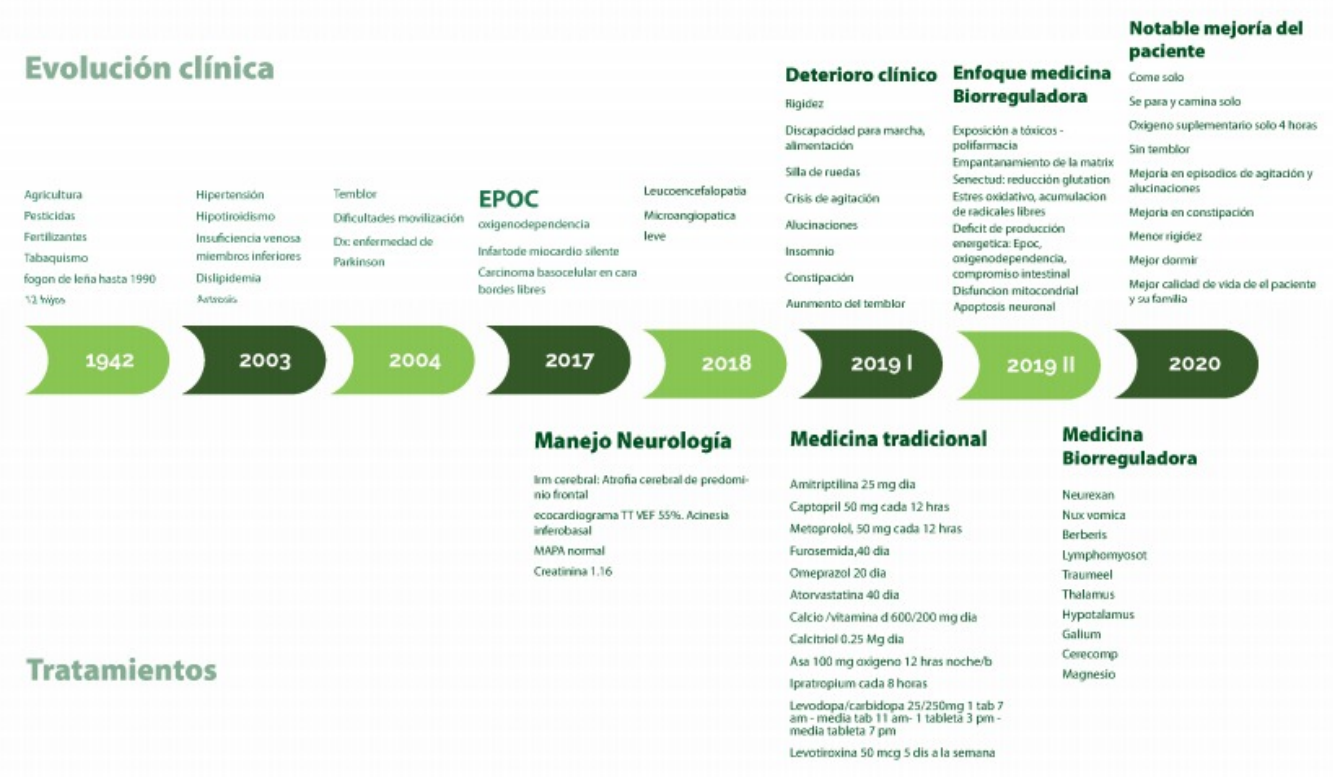

representación esquemática de la línea del tiempo de la enfermedad. 


\begin{tabular}{|c|c|}
\hline Estadio evolutivo & Características \\
\hline Estadio 0 & Ausencia de signos de la enfermedad \\
\hline Estadio 1 & Enfermedad unilateral \\
\hline Estadio 2 & $\begin{array}{l}\text { Enfermedad bilateral con sintomatologia leve, sin trastornos } \\
\text { posturales }\end{array}$ \\
\hline Estadio 3 & $\begin{array}{l}\text { Enfermedad bilateral con sintomatología leve - moderada y trastornos } \\
\text { posturales }\end{array}$ \\
\hline Estadio 4 & $\begin{array}{l}\text { Incapacidad importante derivada de la enfermedad, pero aún es } \\
\text { capaz de caminar de manera independiente }\end{array}$ \\
\hline Estadio 5 & Incapacidad para moverse de manera independiente \\
\hline
\end{tabular}

ESCALA DE HOEHN Y YAHR

estadios componentes de la escala de hoehn y Yahr para calidad de vida. 


\begin{tabular}{|c|c|}
\hline Actividad & Descripción de la dependencia \\
\hline Bañarse & $\begin{array}{l}\text { Independiente: Necesita ayuda para lavarse una parte del cuerpo, } \\
\text { o lo hace solo. } \\
\text { Dependiente: Incluye la necesidad de ayuda para entrar o salir de } \\
\text { la bañera }\end{array}$ \\
\hline Vestirse & $\begin{array}{l}\text { Independiente: Se viste totalmente (incluye coger las prendas del } \\
\text { armario) sin ayuda. Excluye el atado de los cordones de los } \\
\text { zapatos. } \\
\text { Dependiente: No se viste solo }\end{array}$ \\
\hline Usar el retrete & $\begin{array}{l}\text { Independiente: No precisa ningún tipo de ayuda (Incluye la } \\
\text { entrada y salida del baño). Usa el baño. } \\
\text { Dependiente: Incluye usar orinal o cuña. }\end{array}$ \\
\hline Movilidad & $\begin{array}{l}\text { Independiente: No requiere ayuda para sentarse o acceder a la } \\
\text { cama } \\
\text { Dependiente }\end{array}$ \\
\hline Continencia & $\begin{array}{l}\text { Independiente: Control completo de la micción y defecación } \\
\text { Dependiente: Incluye control parcial o total mediante enemas, } \\
\text { sondas o empleo reglado del orinal o cuña }\end{array}$ \\
\hline Alimentación & $\begin{array}{l}\text { Independiente: Leva la comida del plato, o equivalente, a la boca } \\
\text { sin ayuda } \\
\text { Dependiente: Incluye no comer y la nutrición parenteral o enteral } \\
\text { por sonda }\end{array}$ \\
\hline \multicolumn{2}{|c|}{$\begin{array}{l}\text { Por ayuda se entiende la supervisión, dirección o ayuda personal activa: La evaluación } \\
\text { debe realizarse según lo que el enfermo realice y no sobre lo que seria capaz de realizar. }\end{array}$} \\
\hline \multicolumn{2}{|c|}{$\begin{array}{l}\text { Clasificación: } \\
\text { A Independiente en todas las actividades } \\
\text { B Independiente en todas las actividades, salvo una } \\
\text { C Independiente en todas las actividades, excepto bañarse y otra función adicional } \\
\text { D Independiente en todas las actividades, excepto bañarse, vestirse y otra función adicional } \\
\text { E Independiente en todas las actividades, excepto bañarse, vestirse, uso del retrete y otra función } \\
\text { adicional } \\
\text { F Independiente en todas las actividades, excepto bañarse, vestirse, uso del retrete, movilidad y } \\
\text { otra función adicional } \\
\text { G Dependiente en las } 6 \text { funciones }\end{array}$} \\
\hline
\end{tabular}

\section{INDICE DE KATS}

indice o escala de Katz para evaluar independencia de un enfermo para realizar las actividades de la vida que se muestran en la imagen, el puntaje se obtiene por calculadoras en red disponibles. 


\section{Puntuación antes de la intervención}

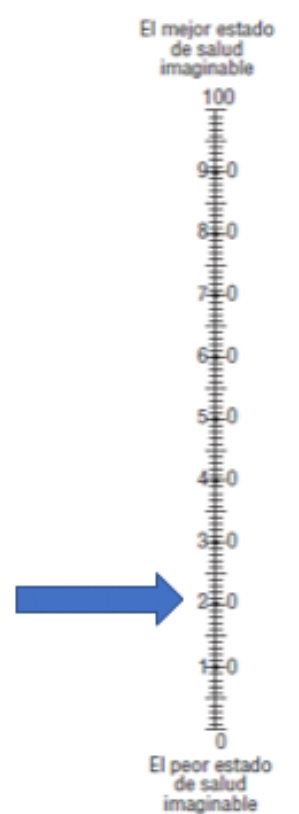

Puntuación despues de

laintervención

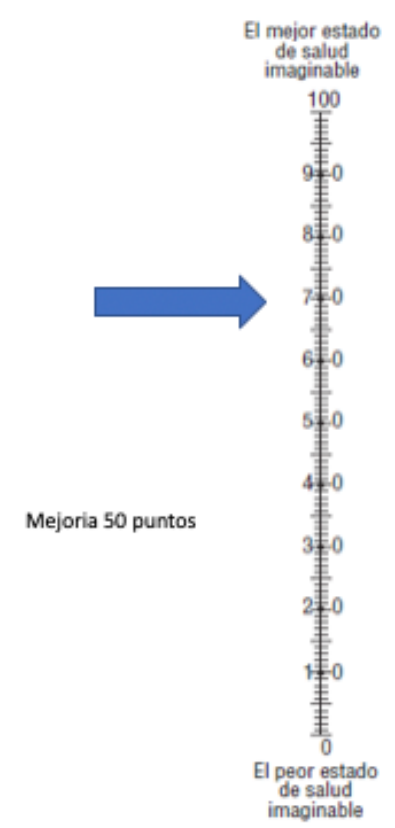

representación grafica del termómetro de calidad de vida de Euroqol 5D: antes de la intervención 20 puntos \% ; después de la intervención 70 puntos \% . 


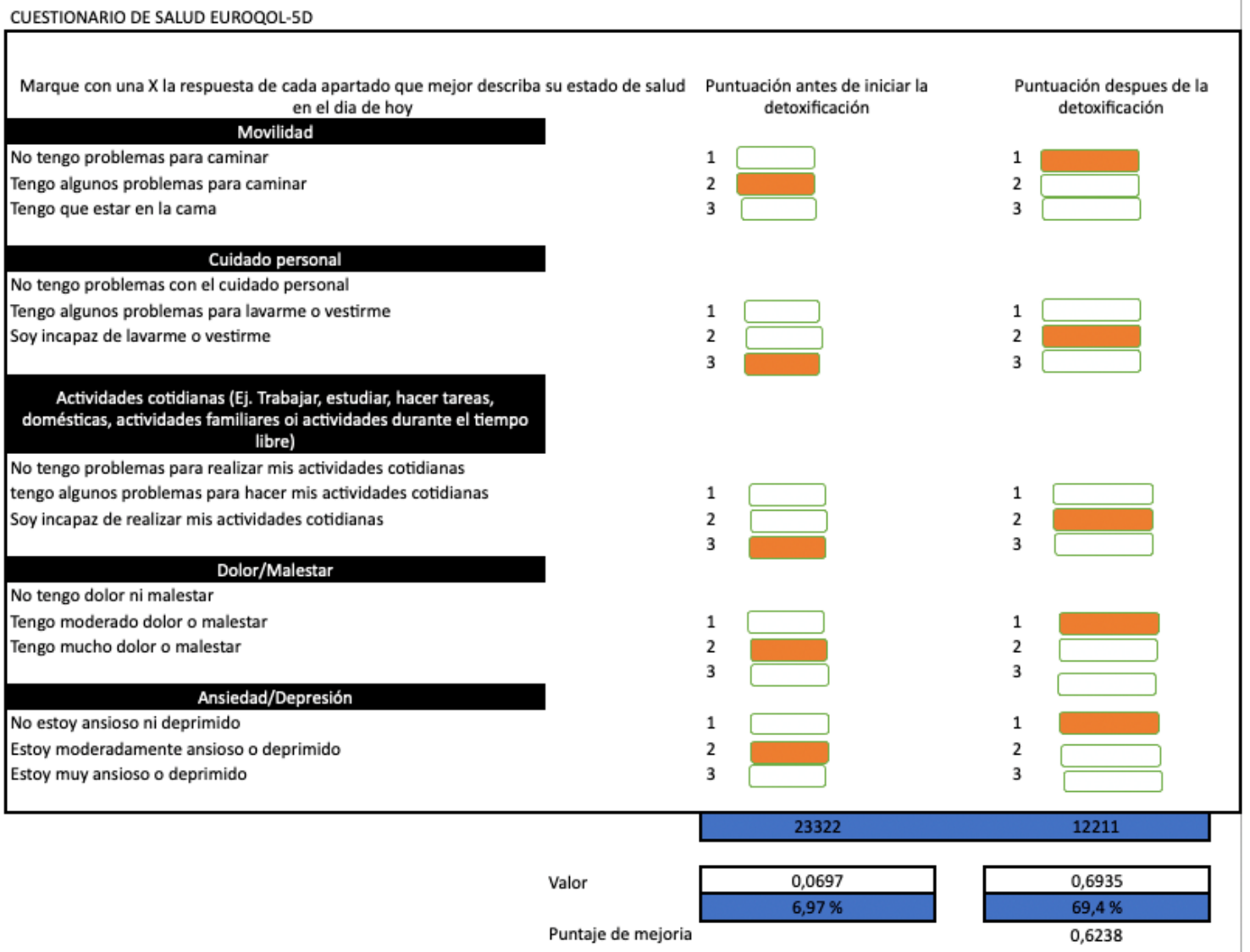

representación grafica de escala de calidad de vida de Euroqol 5D : antes de la intervención 6.97\%, después de la intervención $69.4 \%$. 